\title{
Interactive comment on "Statistical Hypothesis Testing in Wavelet Analysis: Theoretical Developments and Applications to India Rainfall" by Justin A. Schulte et al.
}

\section{Anonymous Referee \#2}

Received and published: 18 March 2019

General comments: The paper highlights (and provides the solution to) an important aspect of the application of wavelet analysis which is relevant to the broad field of geophysics and beyond, where an understanding of non-linear and complex processes is required. Correlation among wavelet coefficients is a very well-known issue which has been discussed in the context of forecasting applications using wavelet-based models in many previous studies. However, it is rather rare to see the accommodation of this aspect in wavelet power spectrum and coherence based studies which the author has successfully demonstrated in this paper.

The author provides a novel method of estimating significant periodicities while tacking 
the issue of false-positive results employed in differentiating the significant periodicities in the wavelet power spectrum compared to the background noise of the spectrum. The $R$ package for this application would be very useful to the community and I would strongly recommend the community to use, test and validate the proposed approach. The paper is very well written and provides sufficient details and arguments in support of the study. I would recommend accepting this paper pending some very minor corrections which I have listed below:

Technical corrections:

Many sentences are too terse. Especially in the abstract. For eg: "The output of a recently developed cumulative area-wise......." "Statistical hypothesis tests in wavelet analysis are reviewed and developed.: As there may be multiple kinds of analysis pertaining to the application of wavelets, I would suggest specifying what kind of hypothesis test is discussed in that kind of wavelet analysis in this study.

P2 Line 2-3: "To make such comparisons, one must implement statistical tests." Please specify which tests.

P2 Line 11-12: "the first of which is that the test will frequently generate many false positive results because of the simultaneous testing of multiple hypotheses." Please provide relevant references for this point.

P3 Line 3: "a first survey of the theoretical"- I suggest replacing "survey" with "review".

P3 Line 28: what is the significance or the Normalization of the reproducing kernel. are the mathematically different from that shown in Eq 2? Please clarify.

P5 Line 19: Adjective "concrete" is not required.

Interactive comment on Nonlin. Processes Geophys. Discuss., https://doi.org/10.5194/npg2018-55, 2018. 